\title{
Gain enhancement for wideband end-fire antenna design with artificial material
}

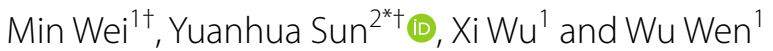

\author{
${ }^{*}$ Correspondence: \\ sunyuanhua17@hotmail.com \\ ${ }^{\dagger}$ Min Wei and Yuanhua Sun \\ contributed equally \\ to this work \\ ${ }^{2}$ The College of Computer \\ Science, Neijiang Normal \\ University, Dongtong Road, \\ Neijiang 641100, China \\ Full list of author information \\ is available at the end of the \\ article
}

\begin{abstract}
Gain enhancement wideband end-fire antenna is proposed in this paper. The proposed antenna can achieve gain enhancement by loading novel artificial materials structures (Split-ring Resonators) in the end-fire direction while broad bandwidth is realized by using elliptic dipole elements and a microstrip to coplanar balun. The measurements show that the proposed antenna have around $5-8 \mathrm{~dB}$ gain in the working band (5-11 GHz), which is around $2 \mathrm{~dB}$ more than the unloaded one. This antenna can be used in target recognition systems for its advantages of end-fire radiation broad bandwidth and high gain.
\end{abstract}

Keywords: Wideband, End-fire antenna, Gain enhancement, Split-ring resonators, Artificial material

\section{Background}

Recently, antennas are much demanded such as high gain, broad bandwidth and endfire radiation patters with the development of military technology, especially in the field of target recognition. Meanwhile, the artificial materials because of its excellent electromagnetic characteristics have been widely used in antenna design (Caloz and Itoh 2005). Artificial materials composed by periodic structures such as split-ring resonators (Pendry et al. 1999), complementary split-ring resonators (Baena et al. 2005), composite right/left-handed transmission lines (CRLH TLs) (Lai et al. 2004) and electric LC resonators (Schurig et al. 2006). A microstrip antenna with zero-index materials(ZIM) whose permeability close to zero can achieve 1-2 dB gain improvement (Ma et al. 2009). Great interests have been focused on meta-based antennas. Among them, low/zeroindex meterials (LIM/ZIM) have features of controlling the direction of emission (Lovat et al. 2007; Zhou et al. 2009). In Zhou et al. (2009), with ZIM structure as the superstrate of a microstrip patch antenna, 1-2 dB gain improvement was obtained. However, this kind of structure makes the antenna thick in profile and heavy in weight. In Huang et al. (2008), Huang proposed split-ring resonator(SRR) structure that can be used in the design of antenna. When dipole antenna is brought close to the SRR array, the antenna can achieve around $3 \mathrm{~dB}$ more than the gain of the dipole antenna in free space. However the bandwidth of these antennas are quite narrow.

The bowtie dipoles are widely used for their simple structure and broad bandwidth (Lin and Tsai 1998; Kiminami et al. 2004). In Qu et al. (2008), a wideband periodic

(c) 2016 The Author(s). This article is distributed under the terms of the Creative Commons Attribution 4.0 International License (http://creativecommons.org/licenses/by/4.0/), which permits unrestricted use, distribution, and reproduction in any medium, provided you give appropriate credit to the original author(s) and the source, provide a link to the Creative Commons license, and indicate if changes were made. 
endfire antenna with an impedance bandwidth of $51.4 \%$ and unidirectional patterns was proposed based on the idea of a log-periodic antenna. Nevertheless, its gain with an average of $4 \mathrm{~dB}$ was still low.

In this paper, gain enhancement for wideband end-fire antenna by using split-ring resonator (SRR) structures is designed for target recognition system. The broad bandwidth of the proposed antenna is obtained by using the balun of microstrip-to-slotline transitions. Gain enhancement is achieved by loading two rows of split-ring resonator structures (SRRs) symmetrically along the end-fire direction while maintaining the wideband performance of the proposed antenna. The proposed end-fire antenna that loaded SRRs can obtain $5-8 \mathrm{~dB}$ gain in the whole operating band $(5-11 \mathrm{GHz})$, which is around $2 \mathrm{~dB}$ more than unloaded one. The proposed antenna is simulated by using the high frequency structure simulator (HFSSv15) software based on the finite element method. The measured results of the fabricated antenna show good agreement with the simulated results. The proposed broadband SRRs-loaded antenna with end-fire patterns has more valuable in target recognition systems.

\section{Antenna design}

The proposed wideband end-fire antenna with SRRs and conventional end-fire antenna are shown in Fig. 1. In the design of the proposed antenna, the feed uses microstrip-toslotline transition. The proposed antenna uses the slotline at the bottom to couple the coplanar stripline at the top layer. The circular slots at the bottom layer and microstrip patch at the top layer are used to achieve the required impedance matching across the operating band $(5-11 \mathrm{GHz})$. In the design of the proposed antenna, the directors of the conventional end-fire antenna are replaced by two rows split-ring resonator structures (SRRs) shown in Fig. 1. Details of the SRR unit cell are shown in Figs. 1c and 2. The

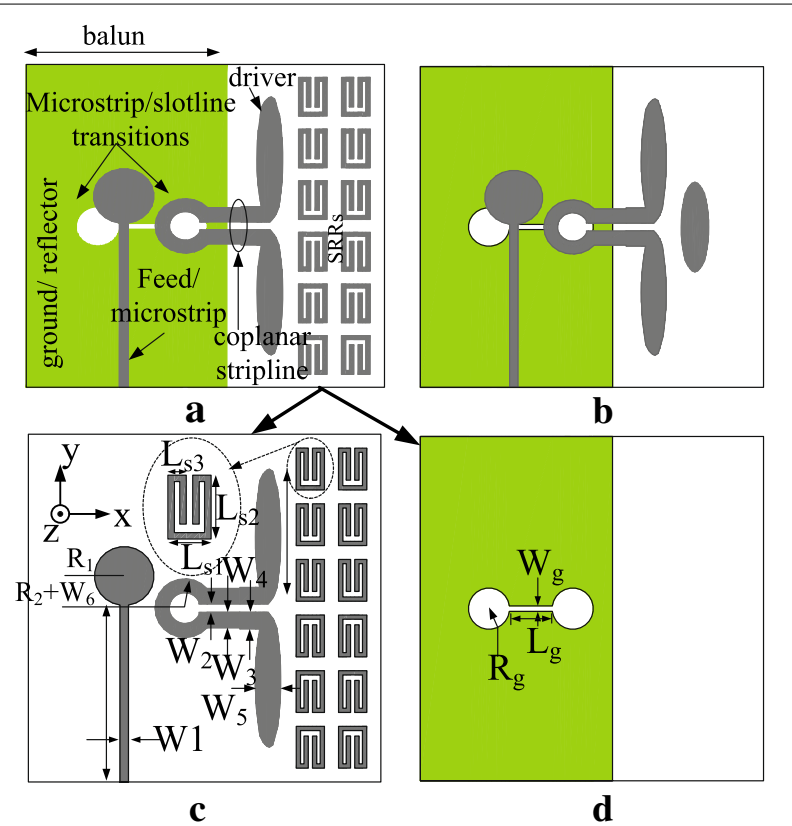

Fig. 1 The proposed antenna a: overall view of proposed antenna; $\mathbf{b}$ : overall view of conventional antenna; $\mathbf{c}$ Top view of the proposed antenna; $\mathbf{d}$ : Bottom view of the proposed antenna 

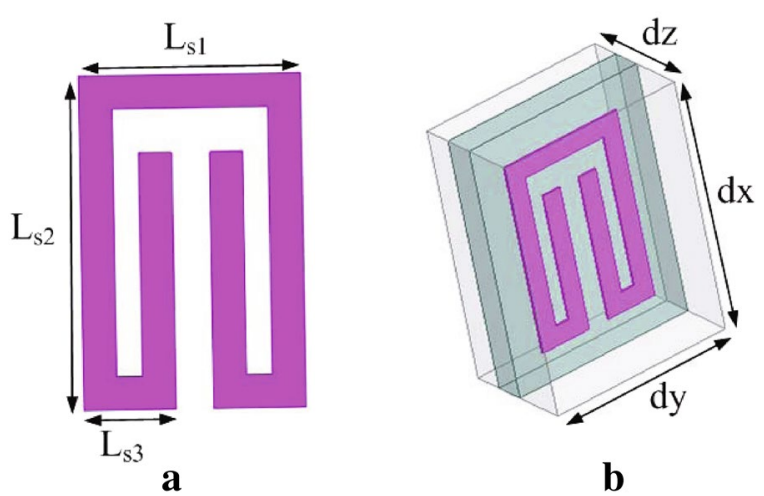

Fig. 2 The proposed SRR unit and simulation model. a Details of the SRR unit cell. b The simulation model

split-ring resonator structure is simple and is easy to get larger capacitance. So It is convenience to tune the resonant frequency of the resonator structure by three main parameters $L_{s 1}, L_{s 2}$ and $L_{s 3}$.

For demonstration purpose in the laboratory, the proposed antenna is designed on a $0.635 \mathrm{~mm}$ Rogers RT 6010 substrate with a dielectric constant $\varepsilon_{r}=10.2$. The proposed wideband end-fire antenna composes of a microstrip-to-coplanar balun, elliptic dipole elements, the metallisation on the bottom plane is a truncated microstrip ground as the reflector and eight SRRs that are printed on the top of the substrate symmetrically in the endfire direction as shown in Fig. 1. All of the proposed antenna geometric parameters are optimised using Ansoft HFSSv15. The dimensions of the proposed antenna are shown in the Table 1.

The SRRs loaded part consists of twelve SRRs which are printed on the surface of the substrate symmetrically in the end-fire direction. Details of the SRR unit cell are described in Fig. 2a. We use single-ring rectangular SRRs instead of dual-ring SRRs for three reasons: (a) Single-ring SRR owns a simple structure and is easy to get larger capacitance; (b) It is more convenience to turn the resonant frequency of the single-ring SRR structure by two main parameters $L_{s 2}$ and $L_{s 3}$; $(c)$ The asymmetric structure of the inner and outer rings in the dual-ring SRR structure contributes gain enhancement for the two opposite directions. However, gain enhancement is better in one direction for the sing-ring SRRs which is a good choice for the proposed periodic end-fire antenna.

Operation principle is analyzed by investigating the current distributions of both the unloaded and loaded antennas which is given in Fig. 3. Comparing the current distributions of the SRR-loaded antenna with the unloaded one,we find that the SRR part

Table 1 The dimensions of the proposed antenna

\begin{tabular}{lllllll}
\hline $\boldsymbol{W}_{\mathbf{1}}$ & $\boldsymbol{W}_{\mathbf{2}}$ & $\boldsymbol{W}_{\mathbf{3}}$ & $\boldsymbol{W}_{\mathbf{4}}$ & $\boldsymbol{W}_{\mathbf{5}}$ & $\boldsymbol{W}_{\mathbf{6}}$ & $\boldsymbol{R}_{\boldsymbol{c}}$ \\
\hline $1.1 \mathrm{~mm}$ & $1.2 \mathrm{~mm}$ & $0.6 \mathrm{~mm}$ & $1.2 \mathrm{~mm}$ & $1.81 \mathrm{~mm}$ & $0.6 \mathrm{~mm}$ & $2.1 \mathrm{~mm}$ \\
\hline $\boldsymbol{R}_{\mathbf{1}}$ & $L_{s \mathbf{1}}$ & $L_{s \mathbf{2}}$ & $L_{s 3}$ & $\boldsymbol{R}_{\boldsymbol{g}}$ & $\boldsymbol{L}_{\boldsymbol{g}}$ & $\boldsymbol{W}_{\boldsymbol{g}}$ \\
\hline $1 \mathrm{~mm}$ & $2 \mathrm{~mm}$ & $3.1 \mathrm{~mm}$ & $0.86 \mathrm{~mm}$ & $1.5 \mathrm{~mm}$ & $3 \mathrm{~mm}$ & $0.41 \mathrm{~mm}$ \\
\hline
\end{tabular}



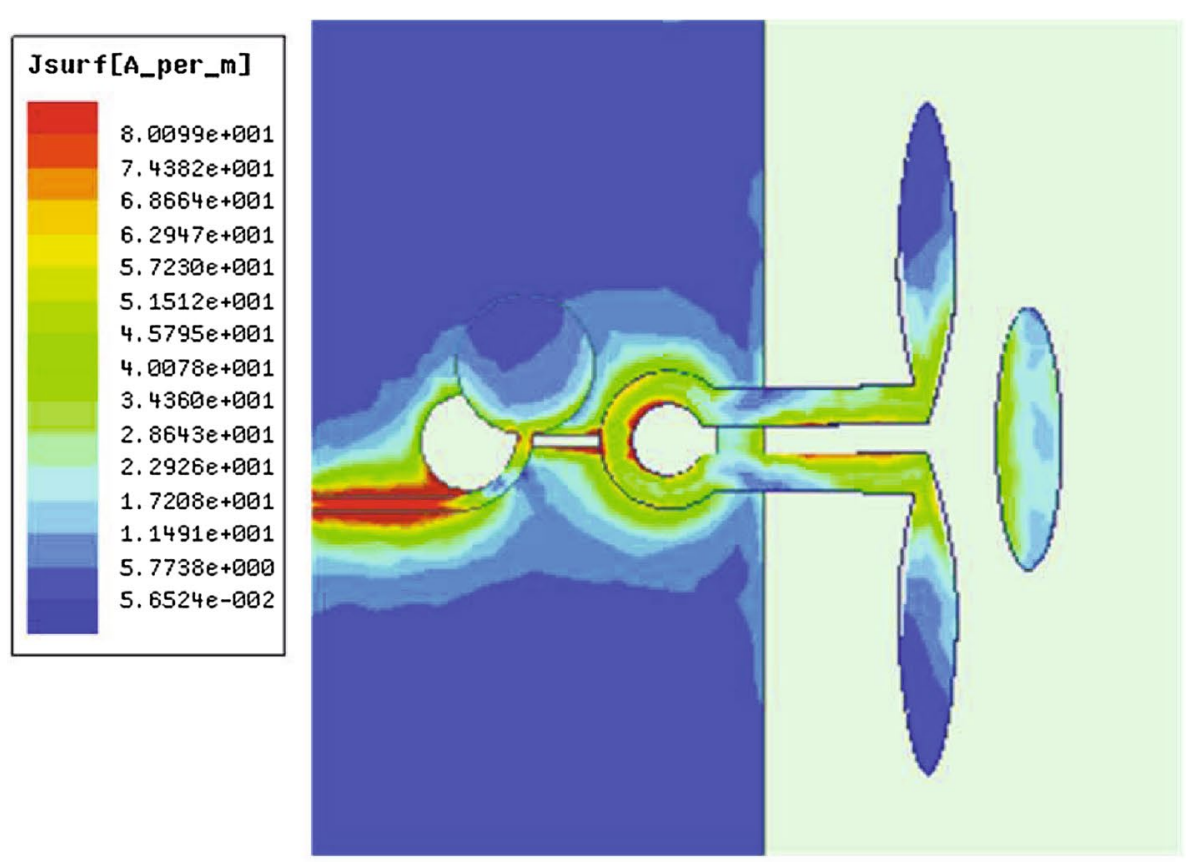

a
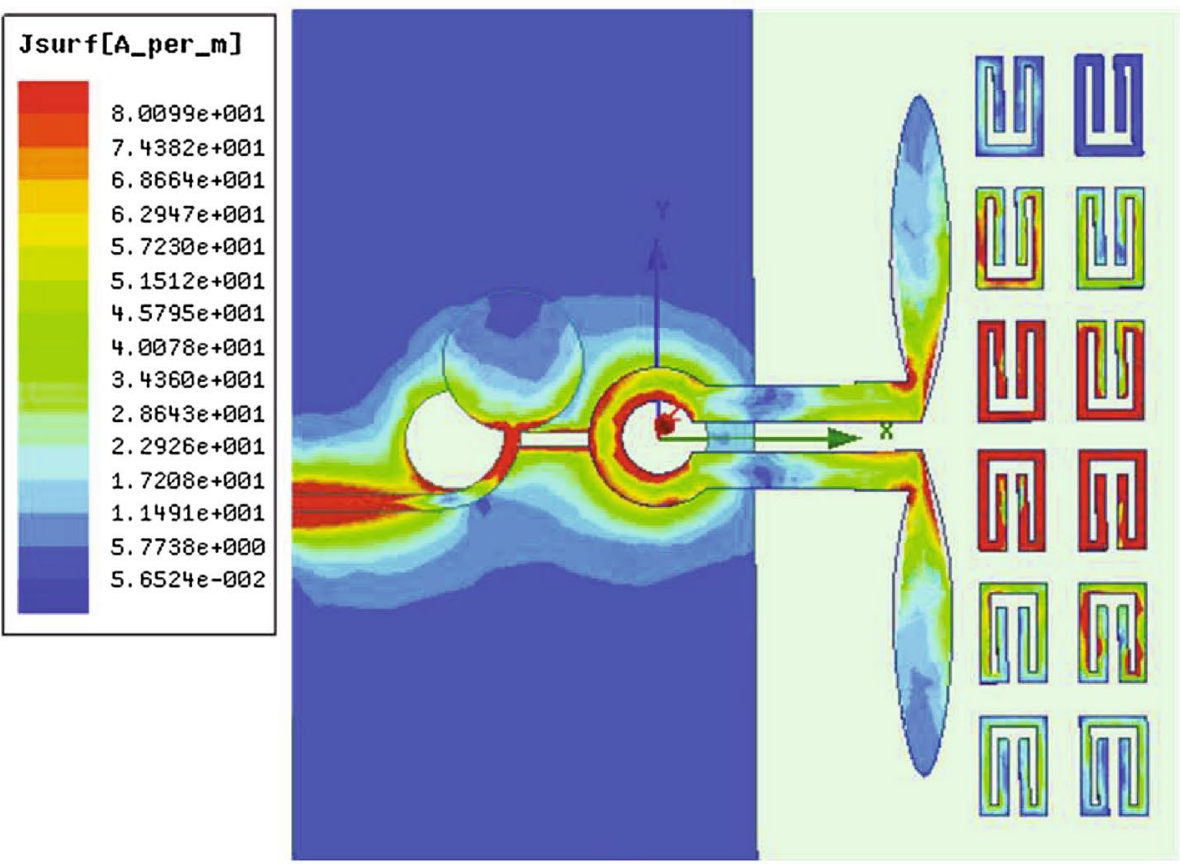

b

Fig. 3 Current distribution of the two antennas; $\mathbf{a}$ antenna without loading structures; $\mathbf{b}$ antenna with SRR loading structures

makes vital contribution on the current movement. Further investigation on the SRR structure is made to find the relationship between gain enhancement and the characteristics of the SRR-loaded part. The most common and effective method to extract 
parameters is given in Chen et al. (2004), Smith et al. (2005). The scattering parameters of the model can be obtained from simulation results of HFSS, and are turned into structural parameters through methematical calculations. Figure $2 \mathrm{~b}$ is simulation model for extracting the effective index of metamaterials. The perfect electric conductor (PEC) and perfect magnetic conductor (PMC) boundary conditions are set in the up-down faces and front-back faces of the box,respectively. The SRR can create the strongest resonance when the E-field is aligned parallel with XY-plane and the $\mathrm{H}$-field is aligned with the center axis(z)of SRR. The dimension of the box are $\mathrm{dx}=4 \mathrm{~mm}$, dy $=3 \mathrm{~mm}, \mathrm{dz}=1.9 \mathrm{~mm}$. The dimension of $\mathrm{dx}$, dy of the simulation configuration which can be considered as the periodicity of the unit cell in the $\mathrm{x}$ - and $\mathrm{y}$-directions respectively, is about the same $\mathrm{s}$ is given in Fig. 1 (which is depicted in dash ellipse). Lager $\mathrm{dx}$ or dy leads to lower resonant frequency, while larger $\mathrm{dz}$ leads to larger resonant frequency.

Both simulated S-parameters (S21) of the SRR unit and retrieved parameters from the simulated S-parameters of the SRR unit are presented in Fig. 5. Four type of loading structures are considered for controlling the performance of the antenna. On the one hand, from Fig. 5a, we can find that SRR metal structure affects the transmission characteristics of the whole loading unit cell. When it is working at the resonant frequency, S21 is so small that little energy can transmit through the substrate with the SRRs and thus the antenna with this loading structure own low gian at resonant frequencies. On the other hand, from Fig. 5b, we can find that when SRR unit cell resonants, the retrieved parameters $(\operatorname{Re}(\mathrm{n}))$ from the simulated S-parameters owns a sudden abrupt change. From snell' $\mathrm{s}$ law $n_{s} \cdot \sin \theta_{1}=n_{0} \cdot \sin \theta_{2}$, which is shown in Fig. 4 . When the refractive index $\left(n_{s}\right)$ of the loading structure gets larger, with the input angle $\theta_{1}$ unchanged, the output angle $\theta_{2}$ get larger, so the energy can congregate according to endfire reference plane (xy-plane). Thus the high gain can be realized in the endfire direction. As shown in Fig. 5b, when the working band of the antenna is higher than the resonant frequency, Type A as an example, the refractive index is lower than that of the substrate (Type D) and thus gain enhancement can be obtained in the whole working band of the antenna. The same conclusion can also be deduced from the transmission characteristics of the loading unit cell which is given in Fig. $5 \mathrm{a}$.

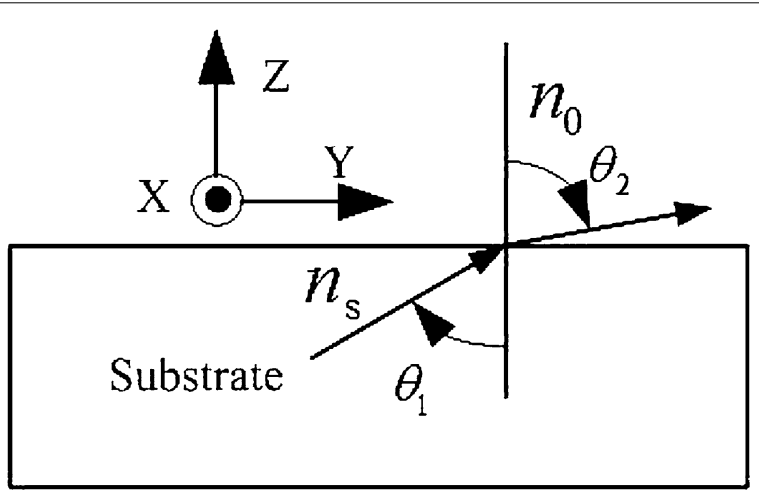

Fig. 4 Electromagnetic wave transmitting sketch map 


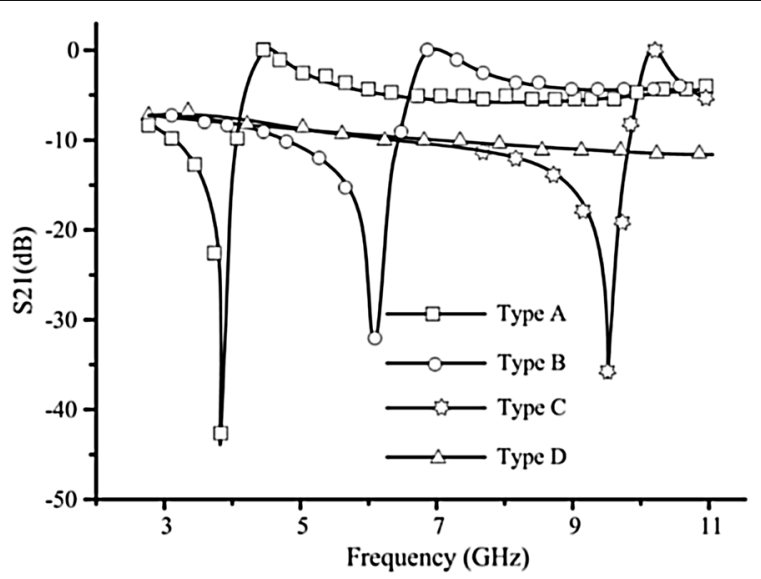

a

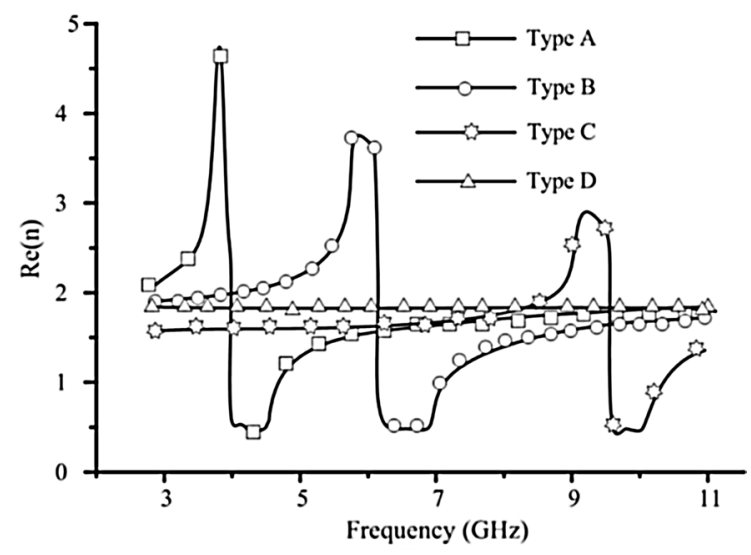

b

Fig. 5 a Simulated S-parameters (S21) of the SRR unit. b Retrieved parameters from the simulated S-parameters of the SRR unit. Type $A: L_{s} 1=2 \mathrm{~mm}, L_{s} 2=3 \mathrm{~mm}, L_{s} 3=0.83 \mathrm{~mm}$. Type $B: L_{s} 1=2 \mathrm{~mm}, L_{s} 2=2.5 \mathrm{~mm}$, $L_{s} 3=0.83 \mathrm{~mm}$. Type $C: L_{s} 1=2 \mathrm{~mm}, L_{s} 2=3 \mathrm{~mm}, L_{s} 3=0.95 \mathrm{~mm}$. Type $D:$ No SRR is loaded, only substrate without copper is used

\section{Measurement results and discussion}

As shown in Fig. 6, A prototype was fabricated to verify the proposed antenna design. All measured results of the proposed antenna were carried out in anechoic chamber using a vector network analyzer (VNA). All simulations were performed by Ansoft high-frequency structure simulation (HFSSv15) based on the finite-element method. As

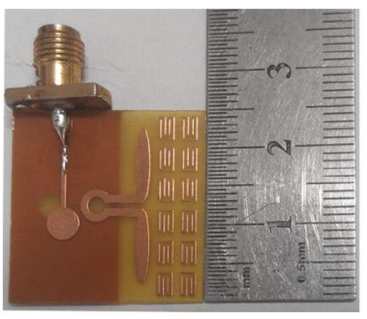

a

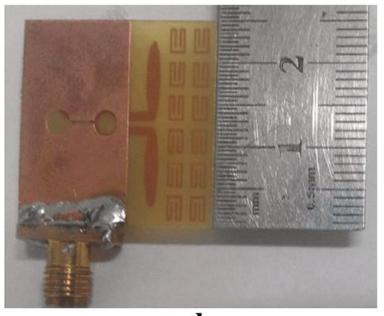

b

Fig. 6 The fabricated proposed antenna a Top view; b Bottom view 
shown in Fig. 7, the measurement results are very good agreement with the simulation results. The proposed antenna can cover wide bandwidth from 5 to $11 \mathrm{GHz}$, the measurement and simulation data of the proposed antenna are in good agreement. Figure 7 shows the proposed antenna can achieve high gain from 6 to $8 \mathrm{~dB}$ in the operating bandwidth from 5 to $11 \mathrm{GHz}$ in the end-fire direction. Figure 7 and Table 2 show the gain of the proposed antenna (loaded SRRs) have $2 \mathrm{~dB}$ more than the gain of the conventional antenna (unloaded SRRs) while the proposed antenna maintaining the wide bandwidth.

The radiation patterns of the proposed end-fire antenna are shown in Fig. 8. The measurement and simulation radiation pattern are in good agreement.It is clear that the proposed antenna has a good end-fire performance while its front-to-back ratio is better than $15 \mathrm{~dB}$ across the $75 \%$ fractional bandwidth. The proposed antenna has a single main beam in the end-fire direction.

\section{Conclusion}

In this letter, a gain enhancement wideband End-fire Antenna has been proposed. The proposed antenna uses split-ring resonators structures (SRRs) and achieves gain enhancement by using the magnetic resonators concept in the antenna design. By substituting the conventional director of the parasitic element in the conventional end-fire antenna with SRRs directions, gain enhancement is achieved in the end-fire direction while maintaining the wideband performance. The measurements show that the SRRsloaded antenna can achieve 5-8 $\mathrm{dB}$ gain in the whole working band (5-11 GHz), which has a fractional bandwidth of $75 \%$, more than $15 \mathrm{~dB}$ front-to-back ratio and around $2 \mathrm{~dB}$ more than the gain of the conventional one that unloaded SRR structures. The proposed

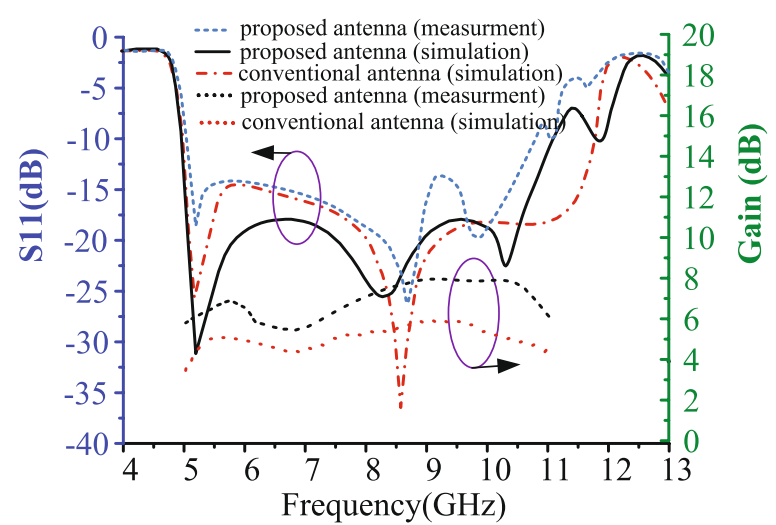

Fig. 7 Simulation and measured return losses and gain for the proposed end-fire antenna and conventional end-fire antenna

Table 2 The Gain of the proposed antenna loaded SRRs and the conventional antenna unloaded SRRs

\begin{tabular}{llllllll}
\hline Antenna & $\begin{array}{l}\mathbf{5 G H z} \\
(\mathbf{d B})\end{array}$ & $\begin{array}{l}\mathbf{6 G H z} \\
(\mathbf{d B})\end{array}$ & $\begin{array}{l}\mathbf{7 G H z} \\
(\mathbf{d B})\end{array}$ & $\begin{array}{l}\mathbf{8} \mathrm{GHz} \\
(\mathbf{d B})\end{array}$ & $\begin{array}{l}\mathbf{9 G H z} \\
(\mathbf{d B})\end{array}$ & $\begin{array}{l}\mathbf{1 0 \mathrm { GHz }} \\
(\mathbf{d B})\end{array}$ & $\begin{array}{l}\mathbf{1 1 \mathrm { GHz }} \\
(\mathbf{d B})\end{array}$ \\
\hline The proposed antenna & 6 & 6.8 & 5.9 & 7 & 8 & 7.9 & 5.9 \\
The conventional antenna & 3.9 & 5 & 4.3 & 5.6 & 6 & 5.5 & 4.3 \\
\hline
\end{tabular}




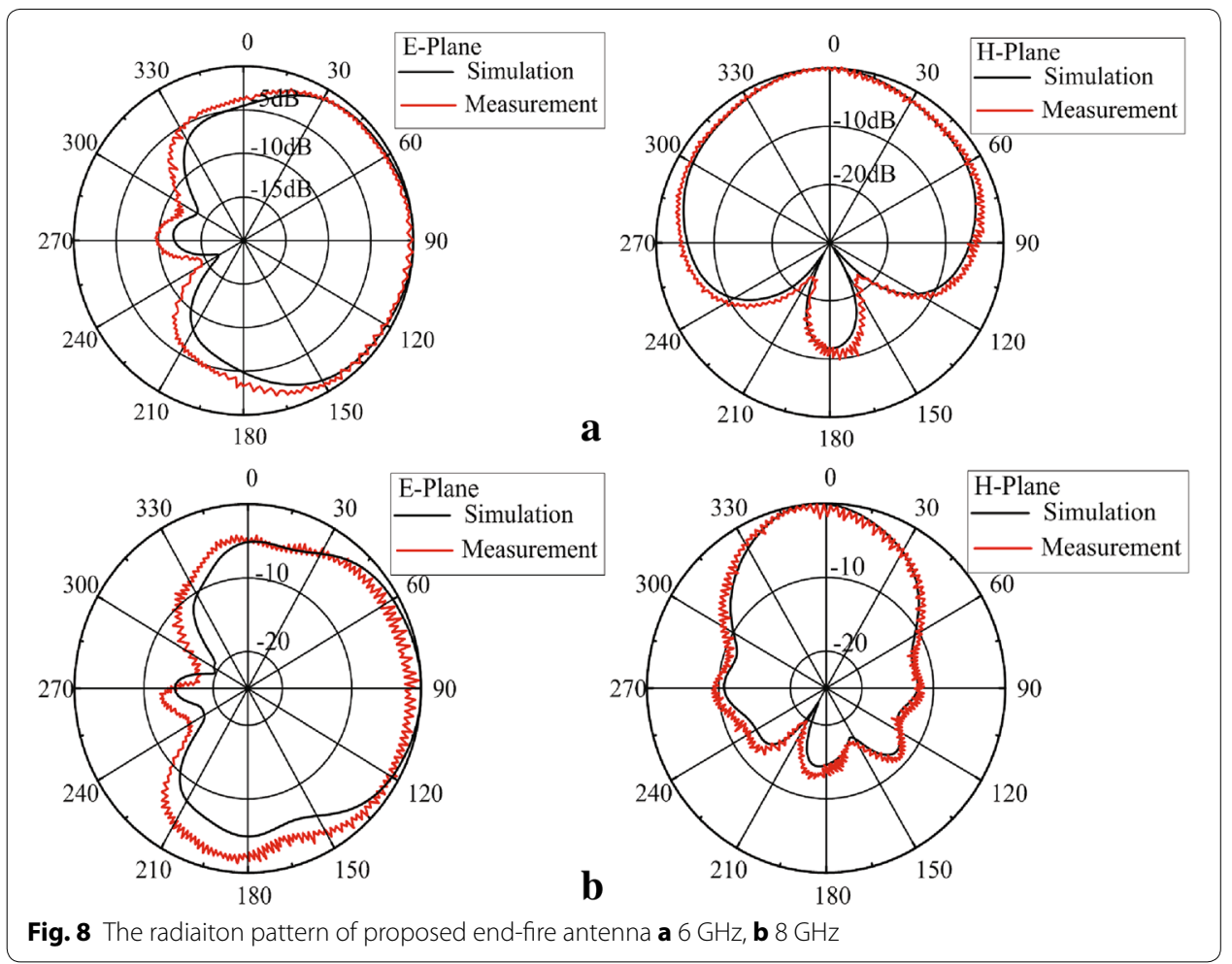

antenna is valuable in target Recognition systems for its advantages of broad band width,high gain and end-fire performance.

\section{Authors' contributions}

WM and SY carried out the studies of artificial material and antenna, and drafted the manuscript. WM is associate professor in Chengdu University of Information Technology and postdoctoral in USA Vanderbilt University, he received the doctor degree from Chinese Academy of Science. SY received the doctor degree in communication engineering from University of Electronic Science and Technology of China in 2015, he is lecturer in Neijiang Normal University. WX and WW carried out the empirical analysis. WX is lecturer in Chengdu University of Information Technology. WW is lecturer in Chengdu University of Information Technology, he received the doctor degree from Chengdu University of Technology. All authors read and approved the final manuscript.

\section{Author details}

${ }^{1}$ The College of Computer Science, Chengdu University of Information Technology, Xuefu Road, Chengdu 610225, China. ${ }^{2}$ The College of Computer Science, Neijiang Normal University, Dongtong Road, Neijiang 641100, China.

\section{Acknowledgements}

We wish to express our gratitude to the referee for his/her invaluable comments and suggestions. This work was supported by Chengdu University of Information Technology.

\section{Competing interests}

The authors declare that they have no competing interests.

Received: 18 March 2016 Accepted: 10 August 2016

Published online: 15 September 2016

\section{References}

Baena JD, Bonache J, Martin F, Sillero RM, Falcone F, Lopetegi T, Laso MA, Garcia-Garcia J, Gil I, Portillo MF et al (2005) Equivalent-circuit models for split-ring resonators and complementary split-ring resonators coupled to planar transmission lines. IEEE Trans Microw Theory Tech 53(4):1451-1461

Caloz C, Itoh T (2005) Electromagnetic metamaterials: transmission line theory and microwave applications. Wiley, New Haven

Chen X, Grzegorczyk TM, Wu B-I, Pacheco J Jr, Kong JA (2004) Robust method to retrieve the constitutive effective parameters of metamaterials. Phys Rev E 70(1):016608 
Huang Y, De A, Zhang Y, Sarkar TK, Carlo J (2008) Enhancement of radiation along the ground plane from a horizontal dipole located close to it. IEEE Antennas Wirel Propag Lett 7:294-297

Kiminami K, Hirata A, Shiozawa T (2004) Double-sided printed bow-tie antenna for UWB communications. IEEE Antennas Wirel Propag Lett 1(3):152-153

Lai A, Itoh T, Caloz C (2004) Composite right/left-handed transmission line metamaterials. IEEE Microw Mag 5(3):34-50

Lin Y-D, Tsai S-N (1998) Analysis and design of broadside-coupled striplines-fed bow-tie antennas. IEEE Trans Antennas Propag 46(3):459-460

Lovat G, Burghignoli P, Capolino F, Jackson D (2007) Combinations of low/high permittivity and/or permeability substrates for highly directive planar metamaterial antennas. IET Microw Antennas Propag 1(1):177-183

Ma Y, Wang P, Chen X, Ong C (2009) Near-field plane-wave-like beam emitting antenna fabricated by anisotropic metamaterial. Appl Phys Lett 94(4):044107

Pendry JB, Holden AJ, Robbins D, Stewart W (1999) Magnetism from conductors and enhanced nonlinear phenomena. IEEE Trans Microw Theory Tech 47(11):2075-2084

Qu S-W, Li J-L, Xue Q, Chan C-H (2008) Wideband periodic endfire antenna with bowtie dipoles. IEEE Antennas Wirel Propag Lett 7:314-317

Schurig D, Mock J, Smith D (2006) Electric-field-coupled resonators for negative permittivity metamaterials. Appl Phys Lett 88(4):041109

Smith D, Vier D, Koschny T, Soukoulis C (2005) Electromagnetic parameter retrieval from inhomogeneous metamaterials. Phys Rev E 71(3):036617

Zhou H, Pei Z, Qu S, Zhang S, Wang J, Duan Z, Ma H, Xu Z (2009) A novel high-directivity microstrip patch antenna based on zero-index metamaterial. IEEE Antennas Wirel Propag Lett 8:538-541

\section{Submit your manuscript to a SpringerOpen ${ }^{\circ}$ journal and benefit from:}

- Convenient online submission

\section{- Rigorous peer review}

- Immediate publication on acceptance

- Open access: articles freely available online

- High visibility within the field

Retaining the copyright to your article

Submit your next manuscript at $\boldsymbol{\nabla}$ springeropen.com 